\title{
Peer Support in Improving Self-Efficacy of Rural Patients with Type 2 Diabetes and the Application of Drugs to Reduce the Pain from Diabetes
}

\author{
Ailan Pan, Longbo Liao*, Qiaoyun Li, Liyan Qin \\ The People's Hospital of Lipu County, Guilin, China \\ Email: 1036568905@qq.com, “461970726@qq.com, 342821788@qq.com, 625028544@qq.com
}

Received 20 February 2016; accepted 24 April 2016; published 27 April 2016

Copyright () 2016 by authors and Scientific Research Publishing Inc.

This work is licensed under the Creative Commons Attribution International License (CC BY). http://creativecommons.org/licenses/by/4.0/

\section{(c) (i) Open Access}

\begin{abstract}
Objective: This study aims to explore the effect of peer support in improving self-efficacy of rural patients with type 2 diabetes and the application of drugs to reduce the pain from diabetes. Methods: Patients with type 2 diabetes in the rural area were selected and they were divided into the peer group and control group with voluntary. The patients in peer support group applied six kinds of activity patterns, while the control group was carried out with routine activities. Results: Comparison indexes for the two groups are mastering degree of related knowledge to diabetes, control compliance rate of indicators related to diabetes, and management of diabetes self-efficacy. Comparing with the control group, indexes in peer support group were significantly increased $(\mathrm{P}<$ 0.01). Meanwhile, in the table of diabetes distress, 17 items have been improved and the occurrence rate of painful diabetic decreased significantly $(P<0.01)$. Conclusion: Peer support can improve the rural patients' self-efficacy of type 2 diabetes and decrease patients' pain from type 2 diabetes mellitus.
\end{abstract}

\section{Keywords}

Peer Support, Rural Area, Type 2 Diabetes Mellitus, Diabetic Pain, Self-Efficacy

\section{Introduction}

With the development of living standards of urban and rural residents in China, and the change of diet structure, "Corresponding author.

How to cite this paper: Pan, A.L., Liao, L.B., Li, Q.Y. and Qin, L.Y. (2016) Peer Support in Improving Self-Efficacy of Rural Patients with Type 2 Diabetes and the Application of Drugs to Reduce the Pain from Diabetes. Open Journal of Endocrine and Metabolic Diseases, 6, 135-140. http://dx.doi.org/10.4236/ojemd.2016.64018 
the rate of diabetes incidence in rural areas is rising [1]. In order to treat the patients with diabetes, they have to take long-term medication, control their diet, stick with exercise, and monitor their blood glucose regularly, which are too complex to burden patients' spirit and psychology [2]. Fisher [3] and other scholars carried out a research and found that $70 \%$ of patients with diabetes are under diabetic pain. In recent years, a peak population of this disease has happened in the countryside. That's because people in the rural area are lack of the awareness of self-management and self-management skills to diabetes, resulting in a blood glucose control and occurrence of disease complications. The worst is that it will aggravate the diabetic pain of patients in rural areas. The purpose of this study was to investigate the effect of peer support on the improvement of rural patients with type 2 diabetes pain, and provide a reference for guiding the rural self-management of patients with type 2 diabetes.

\section{Data and Methods}

\subsection{Objects}

100 cases of outpatients with 2 type diabetes treated in Li Town Rural hospital diabetes clinic were selected on January to march 2014. Their ages are between 35 to 65 years old, among which 41 cases are male, 59 cases female. All patients are in line with the classification and diagnostic criteria of diabetes formulated by WH0 in 1999. Meanwhile, the condition of diabetes is above one year, HbALC $>7 \%$. And the incidence of severe cognitive dysfunction was excluded. There are 50 cases who agreed to participate in the peer support group, while 50 cases agreed to participate in the control group. There was no significant difference in age, gender, disease and education level $(\mathrm{P}>0.05)$. Therefore, the indexes in the two groups are comparable.

\subsection{Research Methods}

\subsubsection{Establishment of Diabetic Peer Group}

Patients with type 2 diabetes were selected in Li towns Lipu County, Guilin City, the Guangxi Zhuang Autonomous Region, China. All of them met the criteria of the study and agreed to participate in the peer support group with voluntary. They were divided into five groups, 8 to 12 patients for each group. And all the patients signed the informed consent. The crew requirements for the peer support groups are as follows. Team members should agree to accept leader's leadership in peer support group and actively participate in group activities, as well as actively participate in learning knowledge to diabetic. As for selection of leader, those who would be the priority selection are between 35 - 65 years old. And their medical history of diabetes is more than 1 year, with plenty of time and energy to serve the crew. Those who got insulin injection should also be considered first.

\subsubsection{Leader Training}

The training to leader in the peer support groups is relatively difficult, because people in the rural area have low level knowledge related to diabetes and are lack of skills to managing diabetes. 5 volunteers of medical staff in our hospital who got the certificate of health management division were selected to train the leaders in the peer support groups. Here are the training contents: introduction to peer support project, responsibility of leaders, basic diabetes knowledge, inspection of blood glucose, insulin injection technology, use of portable blood glucose meter, effective communication and psychological dredge, etc. The training time lasted for 3 months. At the end of the assessment, the eligible leaders would get the letter of appointment of "diabetes peer support group leader”. And the internship of those qualified leaders lasted for 3 months. With the help of the health managers, the leaders put what they learned in the training.

\subsubsection{Peer Group Activities}

After training, the leaders organized activities for the group members once for half a month. The leaders had to guide group members monthly to attend lectures about knowledge and skills related to diabetes held by the hospital. Secondly, team members exchanged what they learned or shared their own ideas in the meeting exchange. Thirdly, there are outdoor activities for group members. Furthermore, team members got together to watch videos about diabetes. Last but not least, members in the group had to prepare food material to make diabetic diet themselves. Patients in the peer group got mutual encouragement, mutual support, and mutual exchange of learning experiences, which can strengthen self-management skills to diabetes, and release diabetes related distress. 


\subsubsection{Control Group}

Members in the control group only participate in the lecture about diabetes related knowledge, and skills once a month.

\subsection{Evaluation Criteria and Observation Index}

\subsubsection{Evaluation Standard of the Diabetes Distress Scale (DDS)}

The Diabetes Distress Scale (DDS) was written by William H. Polonsky and translated by Yang Qing [4] who also tested the internal consistency reliability of the scale, which was 0.953 . And the split half reliability was 0.91 , while the stability coefficient was 0.84 . Scale is divided into four dimensions, emotional burden, pain from physician, pain caused by patterns of life and pain associated with relationships. There are total 17 entries. Each entry adopts linear score method, a maximum of 6 points and a minimum of 1 point. According to the DDS score standard, if the average score is over 3 points in 17 items, this kind of psychological pain is divided into medium level or above, which needs to cause clinical attention. Followed by the scale of the average over 2 points, this psychological pain is divided into light pain levels. The higher the scores, the more painful the psychological pain is. The questionnaires of diabetes related knowledge was designed by the researchers, including diabetic cognitive, diet therapy and exercise therapy, 4 questions for each items, while drugs treatment is 3 questions, self-monitoring 5 questions. There is a total of five dimensions of 20 items. Scores of each item are from 0 to 5. Option of "fully aware" for each remarks five points, while option of "completely unaware" records 0 points. The total score is from 0 to 100 . The self efficacy scale with the Chinese version of the diabetes management self efficacy scale (C-DMSES) was applied in this study. There are a total number of 20 entries in the scale. Each entry is remarked from 0 score of "completely unable to do" to 10 scores of "completely can do" in order, with the score of 0 to 200 points.

\subsubsection{Observation Targets}

Targets before and after in the two groups of diabetes distress, self-efficacy, knowledge of diabetes, fasting blood glucose, blood glucose 2 hours after meal, glycosylated hemoglobin, total cholesterol, weight, systolic blood pressure, diastolic pressure variations were observed.

\subsection{The Use of Statistical Method}

SPSS 18.0 statistical software was used for data analysis and processing. Statistical software of SPSS 17.0 was used to process statistics. Approximate chi square was used to show the comparison of designed level data in the two groups. Measurement data was showed with Mean \pm standard deviation $(\bar{x} \pm \mathrm{s})$, and tested it with t-test, while count data used $x^{2}$ to test. If $P$ is less than 0.05 , it will be considered to be statistically significant.

\section{Results}

\subsection{Comparison of Diabetes Cognitive Knowledge before-after Activities in the Two Groups}

Before the intervention, the total score of diabetes related knowledge in peer group was 51.90, 9.72 more or less, and in control group was 52.74, 8.95 more or less. The difference was not statistically significant. After intervention, the total score of diabetes related knowledge in peer group became 67.44, 6.69 more or less, and in control group became 55.18, 7.69 more or less. The difference was statistically significant $\mathrm{P}<0.05$. The result was showed in Table 1.

\subsection{Improvement of Diabetic Biochemical Indicators before and after Activities}

Before the intervention, there was no significant difference of the biochemical indicators between the peer group and the control group. After intervention, the levels of fasting blood glucose, glucose two hours after meal, glycosylated hemoglobin, total cholesterol and systolic blood pressure were significantly improved in the peer group and control group. The difference was statistically significant $(\mathrm{P}<0.05)$. However, there was no significant difference in body mass index and diastolic blood pressure. For more details, please look at Table 2. 
Table 1. Comparison of diabetes cognitive knowledge before-after activities in the two groups.

\begin{tabular}{cccccc}
\hline Changes of cognitive knowledge & The peer group & The control group & T & P \\
\hline \multirow{2}{*}{ Diabetes cognition } & before & $10.06 \pm 2.41$ & $10.80 \pm 1.98$ & -1.67 & 0.09 \\
& after & $13.54 \pm 1.91$ & $11.34 \pm 1.74$ & 5.99 & 0.00 \\
\multirow{3}{*}{ Diet treatment } & before & $11.32 \pm 2.41$ & $11.24 \pm 2.39$ & 0.16 & 0.86 \\
& after & $13.86 \pm 1.71$ & $11.60 \pm 2.23$ & 5.67 & 0.00 \\
Sport treatment & before & $8.88 \pm 2.43$ & $9.20 \pm 2.24$ & -0.68 & 0.49 \\
& after & $13.20 \pm 2.06$ & $9.74 \pm 2.10$ & 8.30 & 0.00 \\
Drugs treatment & before & $9.90 \pm 2.23$ & $9.90 \pm 2.25$ & 0.00 & 1.00 \\
& after & $10.84 \pm 1.62$ & $10.12 \pm 1.91$ & 2.03 & 0.04 \\
Self-motoring & before & $11.74 \pm 3.78$ & $11.58 \pm 3.64$ & 0.21 & 0.83 \\
& after & $16.08 \pm 2.26$ & $12.38 \pm 3.09$ & 6.81 & 0.00 \\
Total Scores & before & $51.90 \pm 9.72$ & $52.74 \pm 8.95$ & -0.44 & 0.65 \\
& after & $67.44 \pm 6.69$ & $55.18 \pm 7.69$ & 8.49 & 0.00 \\
& & $<0.01$ & $>0.05$ & - & - \\
\hline
\end{tabular}

Table 2. Improvement of diabetic biochemical indicators before and after activities.

\begin{tabular}{cccccc}
\hline Changes of before-after biochemical indicators & The peer group & The contract group & T & P \\
Fasting blood glucose & before & $9.60 \pm 4.27$ & $8.34 \pm 2.52$ & 1.78 & 0.07 \\
& after & $6.23 \pm 1.10$ & $7.50 \pm 1.60$ & -4.59 & 0.00 \\
Glucose two hours after meal & before & $13.70 \pm 4.78$ & $14.24 \pm 4.94$ & -0.55 & 0.58 \\
Glycosylated hemoglobin & after & $9.74 \pm 1.57$ & $11.82 \pm 2.66$ & -4.76 & 0.00 \\
& before & $8.01 \pm 1.65$ & $8.06 \pm 1.52$ & -0.15 & 0.87 \\
Total cholesterol and systolic blood pressure & after & $6.22 \pm 1.27$ & $6.86 \pm 1.23$ & -2.55 & 0.01 \\
& before & $5.53 \pm 1.68$ & $6.22 \pm 1.69$ & -2.04 & 0.04 \\
Systolic pressure & after & $5.12 \pm 1.11$ & $5.90 \pm 1.57$ & -2.87 & 0.00 \\
& before & $137.16 \pm 18.41$ & $137.34 \pm 21.43$ & -0.04 & 0.96 \\
Diastolic blood pressure & after & $132.08 \pm 13.62$ & $138.76 \pm 17.38$ & -.13 & 0.03 \\
& before & $81.04 \pm 11.22$ & $81.94 \pm 9.30$ & -0.43 & 0.66 \\
Body mass index & after & $81.76 \pm 17.43$ & $83.90 \pm 9.17$ & -44.76 & 0.44 \\
& before & $23.91 \pm 4.56$ & $24.17 \pm 3.43$ & -0.31 & 0.75 \\
& after & $26.52 \pm 1.79$ & $24.45 \pm 2.00$ & -1.79 & 0.07
\end{tabular}

\subsection{Improvement of Diabetes Self-Efficacy and Pain of Diabetes before and after the Activities in the Two Groups}

Before the intervention, the self efficacy of diabetes in peer group was 91.80, 18.40 more or less, while in the control group, it was 97, 26 more or less. The difference was not statistically significant. After the intervention to peer group and control group, diabetes self-efficacy became 150.4, 24 more or less, and 97.20, 19.60 more or less respectively in the two group. There was statistically significant difference $(\mathrm{P}<0.05)$. Before the intervention, incidence rates of diabetic pain in the peer group and control group were $80 \%$ and $78 \%$ respectively. The difference was not statistically significant. Nevertheless, after the intervention, incidence rates of diabetic pain in the peer group and control group turned to be $52 \%$ and $72 \%$ respectively. The difference was statistically significant $(\mathrm{P}<0.05)$. Please look at Table 3.

\section{Discussion}

Peer support is a measure of support from others. People in it have similar experience to life, so as to provide practical experience and knowledge of disease management for others [5].

\subsection{Functions of Peer Support to Improve the Diabetes Knowledge of Patients with Type 2 Diabetes in Rural Area}

Mastering the knowledge of diabetes is the fundamental self-management for diabetic patients. At present, current situation of patients with type 2 diabetes mellitus mastering diabetes related knowledge in rural area can be 
Table 3. Changes of diabetes self-efficacy and pain of diabetes before and after the activities in the two groups.

\begin{tabular}{|c|c|c|c|c|c|}
\hline \multicolumn{2}{|c|}{ Changes of the pain before-after intervention } & \multirow{2}{*}{$\begin{array}{l}\text { The peer group } \\
91.80 \pm 18.40\end{array}$} & \multirow{2}{*}{$\begin{array}{c}\text { The control group } \\
97.00 \pm 26.00\end{array}$} & \multirow{2}{*}{$\begin{array}{c}\mathrm{T} \\
-1.24\end{array}$} & \multirow{2}{*}{$\begin{array}{c}\mathrm{P} \\
0.21\end{array}$} \\
\hline & before & & & & \\
\hline Selt-efficacy & after & $150.4 \pm 24$ & $97.20 \pm 19.60$ & 12.10 & 0.00 \\
\hline \multirow{2}{*}{ Burden of Emotion } & before & $2.38 \pm 0.96$ & $2.34 \pm 1.25$ & 0.14 & 0.89 \\
\hline & after & $1.62 \pm 0.45$ & $2.42 \pm 0.93$ & -5.44 & 0.00 \\
\hline \multirow{2}{*}{ Pain related to Doctors } & before & $1.78 \pm 0.69$ & $1.97 \pm 1.95$ & -1.25 & 0.21 \\
\hline & after & $2.63 \pm 1.45$ & $2.13 \pm 1.02$ & 2.65 & 0.00 \\
\hline \multirow{2}{*}{ Pain concerned with daily routine } & before & $3.09 \pm 0.74$ & $2.86 \pm 0.66$ & 1.51 & 0.13 \\
\hline & after & $1.64 \pm 0.48$ & $2.67 \pm 0.56$ & -9.82 & 0.00 \\
\hline \multirow{2}{*}{ Pain related to interpersonal relationship } & before & $2.00 \pm 1.17$ & $1.87 \pm 1.03$ & 0.55 & 0.57 \\
\hline & after & $1.40 \pm 0.54$ & $1.88 \pm 0.95$ & -3.09 & 0.00 \\
\hline \multirow{2}{*}{ Total rate of diabetes pain } & before & $80 \%$ & $78 \%$ & 0.06 & 4.244 \\
\hline & after & $52 \%$ & $72 \%$ & 0.806 & 0.039 \\
\hline
\end{tabular}

betrayed as follows. First of all, blood glucose monitoring is not standardized. Secondly, they don't balance their eating habit or control their diet blindly. Furthermore, they are lack of understanding of doing sports, because they don't know it's also a treating method to diabetic. They consider that doing exercise can be replaced by doing housework or labor. They do not understand the symptoms of low blood sugar and its protection methods. The majority of patients, they neither even realize hypoglycemia, nor know how to prevent it. The leaders in peer support team organize team members to learn diabetes knowledge in hospital together, and then members in each peer group has to strengthen the knowledge after learning together. The results of this study indicate when comparing the diabetes related knowledge in peer group with the control group, there is oblivious statistical significance $(\mathrm{P}<0.01)$. Scoring rate in peer group is $67.44 \%, 6.69 \%$ more or less, which is higher than that in the study related to diabetes related knowledge carried out by Wang and other scholars in Yinchuan, China [6]. That is $40 \%$. Therefore, a conclusion can be drawn that the function of peer support group can improve the cognition of diabetes related knowledge for rural patients with type 2 diabetes mellitus, through organizing patients with type 2 diabetes mellitus to learn knowledge concerned with diabetes.

\subsection{Functions of Peer Support to Improve the Blood Glucose and Other Biochemical Indicators of Patients with Type 2 Diabetes in Rural Area}

Treatment of patients with type 2 diabetes is a life-long treatment. In the whole process, patients need to pay attention to scientific diets, regular exercise, correct medication, standard insulin injection, positive attitude and happy mood. Only by doing so, can the blood glucose and other related biochemical indicators be well controlled. In the process of peer support, it requires patients to support each other as well as strong will. With the help of peer, patients can put knowledge into practice. For rural patients with type 2 diabetes in the peer group, they can encourage each other, and mutual support can enhance their compliance. From Table 2, it can illustrates that there is great improvement of the peer group of patients with fasting blood glucose, blood glucose two hours after meal, glycosylated hemoglobin, total cholesterol and systolic blood pressure. Comparing with the control group, there is significant difference in the peer group $(\mathrm{P}<0.01)$. But the improvement of diastolic blood pressure is not obvious. The reason is that diastolic blood pressure is in normal level before and after activities. Meanwhile, and the age of patients has much to do with arterial vascular elasticity. That the improvement of body mass index is not ideal may be related to not enough exercise and inadequate diet control.

\subsection{Functions of Peer Support to Improve the Self-Efficacy and Reduce the Pain of Diabetes of Patients with Type 2 Diabetes in Rural Area}

Self efficacy is the core concept of Bandura's social cognitive theory [7], which refers to the judgment of the individual's ability to complete a particular task. Due to the low economic and culture level in rural areas, grasping diabetes knowledge and self-management skills becomes certain difficult for patients, resulting in lower diabetic management of self-efficiency. That's the reason why patients worry a lot about the disease management, treated ways, emotional burden and emotional support, resulting in excessive anxiety and loss of confidence in 
their life. Another main function of peer support is to provide social with emotional support. Peer group members can carry out life practice and attain mutual assistance in social and emotional (psychological) aspects. Scores on self-efficacy the peer group and control group were 150.4, 24 more or less, 97.20, 1.96 more or less respectively. The difference between the two groups is significant. Patients in the peer groups showed a high level of self-efficacy. The incidence rate of patients' pain reduces from $80 \%$ to $52 \%$. Comparing with the control group, there were significant differences in the peer group, which is consistent with the result of the research operated by Han Wenling and other scholars [8].

\section{Conclusion}

Better education effect of diabetes self-management of patients with type 2 diabetes has been presented during peer support educational activities. Comparing with the control group, as to the indexes of self-efficacy, blood biochemical index and cognitive knowledge related to diabetes and diabetes distress's occurring rate before and after the activity in the peer group, there was a statistically significant difference $(\mathrm{P}<0.05)$. Peer support can be used as an effective model for the diabetes management of patients with type 2 diabetes mellitus in rural areas, which has important clinical significance. Although certain achievement has been reached, there are still certain limitations which call further research.

\section{Limitations of the Study}

Some limitations exist in the research, such as relatively short time in the study few selected cases of patients, and some patients unwilling to return to the hospital to reexamine again. Meanwhile, because of the limited funding, the number of selected cases is not enough to attain the representative research results. Therefore, we strongly hope to have more research funding, in order to make the research results more representative.

\section{Acknowledgements}

During this topic research process, we got help from many departments and individuals, such as Yan Li in the Second People's Hospital Guilin City, Guangxi Zhuang Autonomous Region as well as the self funding research project of the Guangxi Zhuang Autonomous Region Health and Family Planning Commission. All of them offered a great support and help in this research. Now here, all of members of this research group show our deepest appreciation to them, and wish them good health and everything goes well.

\section{Fund Project}

The Guangxi Zhuang Autonomous Region Health and Family Planning Commission self funding research projects (project number: Z2014368).

\section{References}

[1] Li, G. (2012) Observation and Analysis on Health Education for Patients with Diabetes in Rural Area. Chinese National Folk Medicine, 21, 75.

[2] Feng, D.Y., Zeng, Z., Liao, F.F., et al. (2010) Analysis on Influenced Factors of Anxiety and Depression to Patients with Type 2 Diabetes Mellitus in Hospital. Chinese Journal of Health Statistics, 27, 129-132.

[3] Fisher, L., Glasgow, R.E., Mullan, J.T., et al. (2008) Development of a Brief Diabetes Distress Screening Instrument. Annals of Family Medicine, 6, 246-252.

[4] Yang, Q., Liu, X.Q., et al. (2010) The Chinese Diabetes Scale to Evaluate Reliability and Validity of Pain. Journal of Nursing, 17, 8-10.

[5] Fisher, L., Skaff, M.M., Mullan, J.T., et al. (2007) Clinical Depression versus Distress among Patients with Type 2 Diabetes. Diabetes Care, 30, 542-548.

[6] Wang, F., Yang, Z.G., Zeng, C.H., et al. (2010) Observation on Diabetes Related Knowledge of Yinchuan Residents. China's Public Health, 26, 1105-1106.

[7] Jaap, V.B., Adavan, P.E. and Lillie, S.B. (1999) The Psychometric Properties of the Diabetes Management SelfEfficacy Scale for Patients with Type 2 Diabetes Mellitus. Journal of Advanced Nursing, 30, 352-359.

[8] Han, W.L., Liu, X.Y., Wang, Y.L., et al. (2013) Analysis on the Relationship between Self-Efficacy and Psychological Distress in Community. Journal of Nursing, 22, 69. 